\title{
Upregulation of the putative oncogene COTE1 contributes to human hepatocarcinogenesis through modulation of WWOX signaling
}

\author{
HAI ZHANG ${ }^{1}$, YUAN TIAN ${ }^{2}$, JIAN SHEN $^{2}$, YUN WANG $^{2}$, YONGHUA XU $^{2}$, \\ YUPING WANG ${ }^{3,4}$, ZEGUANG HAN ${ }^{3}$ and XIANGCHENG $\mathrm{LI}^{2}$
}

\begin{abstract}
${ }^{1}$ Department of Hepatobiliary Surgery, The Affiliated Hospital of Jiangsu University, Zhenjiang $212001 ;{ }^{2}$ Liver Transplantation Center, First Affiliated Hospital of Nanjing Medical University, Key Laboratory of Living Donor Liver Transplantation, Ministry of Public Health, Nanjing 210029; ${ }^{3}$ Shanghai-MOST Key Laboratory for Disease and Health Genomics, Chinese National Human Genome Center at Shanghai, Shanghai 201203; ${ }^{4}$ Laboratory of Medical Biochemistry and Molecular Biology, School of Basic Medical Sciences of Lanzhou University, Gansu 730000, P.R. China
\end{abstract}

Received December 22, 2013; Accepted March 14, 2014

DOI: $10.3892 /$ ijo.2014.2482

\begin{abstract}
Family with sequence similarity 189, also known as COTE1, has been found to be significantly upregulated in hepatocellular carcinoma (HCC) specimens and cell lines and is associated with tumor size and differentiation. Furthermore, COTE1 contributes to hepatocellular carcinogenesis. The overexpression of COTE1 enhanced in vitro cell viability and colony formation in soft agar, and in vivo tumorigenicity of HCC-derived Focus and Huh7 cells. In contrast, COTE1 knockdown via RNAi markedly suppressed these phenotypes in YY-8103 and WRL-68 HCC cell lines. Mechanistic analyses indicated that COTE1 physically associated with WW domain-containing oxidoreductase (WWOX) and modulated WWOX tyrosine phosphorylation. The ectopic overexpression of COTE1 inhibited the WWOX-p53 signaling pathway by reducing the phosphorylation of WWOX at the Tyr33 residue in Focus cells. Conversely, COTE1 silencing activated tyrosine 33 phosphorylation of WWOX and induced WWOX-p53 mediated mitochondrial apoptosis in WRL-68 cells. In addition, COTE1 upregulation in Huh7 cells blocked the WWOX-cyclin D1 pathway via dephosphorylation of WWOX Tyr287, stimulating cell cycle progression whereas phosphorylation of Tyr287 of WWOX induced by COTE1 silencing resulted in activation of WWOX-cyclin D1 signaling, leading to cell cycle arrest in YY-8103 cells. Together, our findings suggest that the cytoplasmic protein COTE1 contributes to HCC tumorigenesis by regulating cell proliferation through the modulation of WWOX signaling.
\end{abstract}

Correspondence to: Professor Xiangcheng Li, Liver Transplantation Center, First Affiliated Hospital of Nanjing Medical University, 300 Guangzhou Road, Nanjing 210029, P.R. China

E-mail:drxcli@njmu.edu.cn

Key words: COTE1, hepatocellular carcinoma, WW domaincontaining oxidoreductase, apoptosis, cell cycle

\section{Introduction}

Hepatocellular carcinoma (HCC) is one of the most fatal tumors worldwide, particularly in Sub-Saharan Africa and Southeastern Asia (1). In recent years, the incidence of HCC in China has increased (2). The major risk factors for the development of HCC include infection by hepatitis B and C viruses, exposure to aflatoxin B1, and cirrhosis of any etiology (3). Liver resection and transplantation are currently regarded the most effective treatments; however, the postoperative survival rate is only $30-40 \%$ at 5 years (4). In addition, most patients with advanced HCC are rejected for treatment because of indications and contraindications of surgery. Therefore, there is an urgent need to advance our understanding of hepatocarcinogenesis and explore novel effective therapeutic strategies.

Family with sequence similarity 189, member B (FAM189B), also called COTE1, maps to chromosome 1q21 and is widely expressed in heart, brain, placenta, lung, liver, skeletal muscle, kidney, and pancreas $(5,6)$. The COTE1 gene was originally identified by Winfield et al, who found that COTE1 is located near the gene for the lysosomal enzyme glucosylceramidase, deficiency of which is associated with Gaucher disease (6). Alternative splicing of COTE1 results in multiple transcript variants: 1,2, and 3. Variant 1 represents the longest transcript and encodes the longest protein (isoform a). Variants 2 and 3 lack an in-frame portion of the 5 ' coding region compared with variant 1 , and the resulting proteins (isoforms $b$ and c) are shorter than isoform a (National Center for Biotechnology Information Reference Sequences). The COTE1 protein contains 669 amino acids with two potential $\mathrm{N}$-glycosylation sites, a leucine zipper, and multiple potential phosphorylation sites and $\mathrm{N}$-myristoylation sites (6). Recent data showed that COTE1 contains a predicted four-transmembrane domain, suggesting that it might reside in a membrane-bound subcellular organelle such as the Golgi (7). Kallin et al found that expression of COTE1 correlated with activation of endogenous SREBP-1 (sterol-regulatory element binding protein) in vitro, and speculated that it plays a role in lipid metabolism (8). Moreover, the protein has been identified 
as a potential binding partner of a $\mathrm{WW}$ domain-containing protein that is involved in tumor suppression $(7,9)$.

In a previous study, we showed for the first time that COTE1 is markedly upregulated in HCC clinical specimens compared with adjacent non-cancerous livers (10). In the present study we verified upregulation of COTE1 in 42 of 80 paired HCC specimens and 11 of 15 HCC cell lines. These findings indicate that COTE1 may represent a new potential oncogene. Subsequent experiments showed that COTE1 contributed to cell growth and colony formation in vitro, and tumorigenesis in vivo. Furthermore, COTE1 was found to physically interact with the tumor suppressor WW domain-containing oxidoreductase (WWOX), blocking its tyrosine phosphorylation and thereby suppressing WWOX-mediated endogenous apoptosis and cell cycle arrest.

\section{Materials and methods}

Tissue specimens. Eighty pairs of clinical specimens were obtained from patients with HCC who were hospitalized in the First Affiliated Hospital of Nanjing Medical University with informed consent. Adjacent non-tumor tissues were excised $2 \mathrm{~cm}$ from the edge of the primary focus. Both HCC specimens and adjacent non-tumor tissues were immediately stored in liquid nitrogen after excision and confirmed by pathological examination. The protocols for investigations involving humans and animals were approved by the Institutional Animal Care and Use Committee at Nanjing Medical University.

Liver cancer cell lines. Human hepatocellular carcinoma cell lines (QGY-7703, Focus, Hep3B, HepG2, HepG2.2.15, Huh7, LM3, LM6, MHCC-H, MHCC-L, PLC/PRF/5, SK-hep-s, SNU-398, WRL-68, and YY-8103) obtained from the Chinese National Human Genome Center at Shanghai were used in this study. All cell lines were propagated at $37^{\circ} \mathrm{C}$ in a $5 \% \mathrm{CO}_{2}$ humidified incubator in Dulbecco's modified Eagle's medium (DMEM) supplemented with $10 \%$ fetal bovine serum, penicillin $(50 \mathrm{U} / \mathrm{ml})$, and streptomycin $(50 \mu \mathrm{g} / \mathrm{ml})$.

Semiquantitative reverse transcription-polymerase chain reaction (RT-PCR) and quantitative real-time PCR. Total RNA was extracted from clinical samples or cell lines using TRIzol solution (Invitrogen) according to the manufacturer's protocol and reverse-transcribed into cDNA using a M-MLV reverse transcriptase kit (Promega). Primers used in semiquantitative RT-PCR and quantitative real-time PCR were as follows: COTE1, 5'-GGGCTCTGACCTAGGCTTCT-3' (forward) and 5'-ACAGAAGCTCTCCCAGTCCA-3' (reverse); $\beta$-actin (loading control): 5'-AGAGCCTCGCCTTTGCCGATCC-3' (forward) and 5'-CTGGGCCTCGTCGCCCACATA-3' (reverse). All primers were synthesized by Shanghai Biosune Co. Ltd.

Immunofluorescence assay. HCC cells grown on polylysinetreated slides were washed twice with phosphate-buffered saline (PBS), fixed with $4 \%$ paraformaldehyde on ice for $30 \mathrm{~min}$, and blocked with 5\% BSA at room temperature. Cells were stained with primary antibody [goat anti-COTE1 antibody (1:50) or mouse anti-WWOX antibody (1:50), Santa Cruz Biotechnology, CA, USA] at $4^{\circ} \mathrm{C}$ overnight, followed by incubation with secondary antibody [Cy5-conjugated anti-mouse secondary antibody (1:200, red); Cy3-conjugated anti-goat antibody (1:200, green), Molecular Probes Inc., Eugene, OR, USA] at room temperature for $30 \mathrm{~min}$. After rinsing three times with PBS-Tween-20, nuclei were stained with 4,6-diamidino-2-phenylindole (DAPI) and the cells were analyzed by inverted fluorescence microscopy.

siRNA preparation. Two siRNAs against COTE1 were designed using the web server of Invitrogen Co. and chemically synthesized by Shanghai GenePharma Co. The commonly used negative control (NC) siRNA supplied by Qiagen was used as a control. The sense and antisense sequences of human COTE1 were as follows: siRNA-2852 sequence: 5'-GUAUGUAAGCCUUCAAUAAdTdT-3' (sense) and 5'-UUA UUGAAGGCUUACAUACdTdT-3' (antisense); siRNA-3129 sequence: 5'-AGCUCUUAACAGUAUGUAAdTdT-3' (sense) and 5'-UUACAUACUGUUAAGAGCUdTdT-3' (antisense).

Construction of COTE1 shRNA plasmid and COTE1 expression vector. To construct RNAi plasmid, the shRNA expression cassette containing sequence identical to the siRNA2852/3129 sequence of the target gene was inserted into the expression plasmid pSUPER containing the polymerase-III H1-RNA gene promoter. Negative control oligos served as controls. For the construction of COTE1 recombinant plasmid, the COTE1 open reading frame was amplified from a human liver cDNA library (Genbank: NM_006589.2) using nested PCR and inserted into pcDNA3.1B-FLAG-GFP (Chinese National Human Genome Center, Shanghai). The sequences of the shRNAs and primers used for COTE1 expression vector construction are shown in Table I.

Cell transfection. RNAi and plasmid transfections were performed using Lipofectamine 2000 (Invitrogen) according to the manufacturer's instructions at a cell density of $30-50 \%$ and $80-90 \%$, respectively.

Cell proliferation and colony formation. For the cell proliferation assay, cells were plated in 96-well plates and cultured for $24 \mathrm{~h}$ to a density of $30-50 \%$ before transient transfection. The cell counting kit-8 (CCK-8; Dojindo Labs.) was used to measure cell viability according to the manufacturer's instructions. Briefly, $90 \mu \mathrm{l}$ DMEM free medium plus $10 \mu \mathrm{l}$ CCK-8 solution was added to the 96 -well plate. After incubation at $37^{\circ} \mathrm{C}$ for $1 \mathrm{~h}$, the absorbance at $450 \mathrm{~nm}$ was measured. Three replicate wells were tested per assay condition, and each experiment was repeated at least three times.

For the colony formation assay, plasmid-transfected cells were plated in $100 \mathrm{~mm}$ plate and cultured for 2-3 weeks until visible colonies were present. Cells were cultured in media containing fetal bovine serum and G418 (Life Technologies, Inc.) at a final concentration of $0.6-1 \mathrm{mg} / \mathrm{ml}$. Colonies were stained with Coomassie Brillant Blue R-250 (CBBR-250) for $1 \mathrm{~h}$. All experiments were repeated independently at least three times.

For the soft agar colony formation assay, 3000 cells were plated in each well of a 24 -well plate containing $1 \%$ base agar and $0.5 \%$ top agar and cultured at $37^{\circ} \mathrm{C}$ for 2-3 weeks. Colonies were counted under a dissecting microscope. All experiments were repeated independently at least three times. 
Table I. Sequences of shRNAs.

\begin{abstract}
Sequences of shRNAs
COTE1-sh2852

Sense GATCCCCGTATGTAAGCCTTCAATAATTCAAGAGATTATTGAAGGCTTACATACTTTTTGGAAA

Antisense AGCTTTTCCAAAAAGTATGTAAGCCTTCAATAATCTCTTGAATTATTGAAGGCTTACATACGGG

\author{
COTE1-sh3129 \\ Sense GATCCCCAGCTCTTAACAGTATGTAATTCAAGAGATTACATACTGTTAAGAGCTTTTTTGGAAA \\ Antisense AGCTTTTCCAAAAAAGCTCTTAACAGTATGTAATCTCTTGAATTACATACTGTTAAGAGCTGGG
}

Sequences of primers used for COTE1 expression vector construction

COTE1-nest-out

Forward GGGTGGAGAGGAGAAAGGAC

Reverse CAGTGCTATAAGAAGGGGCATC

COTE1-nest-in

Forward CGGGATCCACGAGCCCAGTCTCCCGGCTG

Reverse CGGAATTCATGATGCCCTCGCCTAGTGACTCCAGCCGC

Tumor xenograft model. To establish the tumor xenograft model, we first generated stable transfected cell lines. Briefly, Focus and WRL-68 HCC cells were transfected with pcDNA3.1B-cote1Flag and pSUPER-shRNA2852, respectively, and grown in the presence of G418 (0.6-1 mg/ml) for 2-3 weeks. Visible colonies were picked and transferred into 96-well plates with DMEM++ medium and G418. Stable transfected cells in the 96-well plate were digested and successively transferred into 24 -well plates, 6-well plates, and 100-mm plates. Stable cells were injected subcutaneously into both flanks of nude mice (male BALB/c, 4-6 weeks-old). Tumor volume for each mouse was determined by measuring in two dimensions and calculated as: tumor volume $=$ length $\mathrm{x}(\text { width })^{2} / 2$. The tumor tissues were formalinfixed and paraffin-embedded for immunohistochemistry.

Immunohistochemical staining. COTE1 expression in tissues of HCC specimens or nude mouse tumor xenografts was determined by immunohistochemistry. Briefly, formalin-fixed samples were paraffin-embedded and cut into 4- $\mu \mathrm{m}$ sections. Slides were incubated with goat anti-COTE1 polyclonal antibody (1:50; Santa Cruz Biotechnology) or normal goat IgG as a negative control at $4^{\circ} \mathrm{C}$ overnight. For detection, MaxVision ${ }^{\mathrm{TM}}$ HRP-Polymer anti-Goat IHC Kit (Maixin Bio. Ltd., China) was used according to the manufacturer's protocol. Stained slides were observed under a light microscope.

Flow cytometric analysis of cell cycle and apoptosis. Transfected cells were harvested at different time points, fixed in cold $70 \%$ ethanol, washed, rehydrated in PBS, and stained with propidium iodide (PI) binding buffer $(10 \mathrm{mg} / \mathrm{ml}$ RNase A and $10 \mu \mathrm{g} / \mathrm{ml}$ PI) for $30 \mathrm{~min}$ at room temperature. DNA content of the cells was analyzed using a FACSCalibur flow cytometer (BectonDickinson) with collection of 10,000 events. Analyses of cell cycle and apoptotic cells (sub-G1 population) were performed using Becton-Dickinson FACScan.

Terminal deoxyribonucleotide transferase (TdT)-mediated dUTP nick-end labeling (TUNEL) staining. Cells were fixed in
4\% methanol-free formaldehyde solution in PBS ( $\mathrm{pH} 7.4$ ) for $25 \mathrm{~min}$ at $4^{\circ} \mathrm{C}$, washed with PBS for $10 \mathrm{~min}$ at room temperature, and permeabilized in $0.2 \%$ Triton X-100 solution in PBS for further $5 \mathrm{~min}$. After equilibration for $10 \mathrm{~min}$, the cells were incubated with rTdT (Promega) and observed under a fluorescence microscope. A nucleus with bright green fluorescent staining was recorded as a TUNEL-positive event (11).

Western blot analysis. Cell lysates were prepared in cold lysis buffer containing $25 \mathrm{mmol} / 1$ Tris- $\mathrm{Cl}$ ( $\mathrm{pH} 7.5$ ), 5 mmol/1 EDTA, $1 \%$ sodium dodecyl sulfate, and protease inhibitor cocktail (Sigma). After boiling for $5 \mathrm{~min}$, samples were separated by sodium dodecyl sulfate-polyacrylamide gel electrophoresis and transferred onto a polyvinylidene difluoride membrane, which was blocked in $5 \%$ blocking buffer for $2 \mathrm{~h}$ at room temperature. The membrane was incubated with primary antibody in PBS-Tween-20 (0.1\% Tween-20 in PBS) at $4^{\circ} \mathrm{C}$ overnight and with secondary antibody at room temperature for $1 \mathrm{~h}$. Antibodies used in this study were: goat anti-COTE1 (1:200; Santa Cruz Biotechnology), mouse anti-WWOX (1:200; Santa Cruz Biotechnology), rabbit anti-WWOX (phospho Y33, phospho Y287, 1:500; Abcam, UK), mouse anti-flag (1:200; Santa Cruz Biotechnology), rabbit anti-cyclin D1, E1 (1:500; Abcam), rabbit anti-p53 (p53 and phospho S46, 1:500; Abcam), rabbit anti-Bcl-2 (1:500; Abcam), rabbit anti-caspase 3, 9 (1:500; Abcam), and anti- $\beta$-actin (1:500; Santa Cruz Biotechnology). Proteins were detected using the Odyssey Infared Imaging System (Li-COR).

Co-immunoprecipitation. Cells transfected with pcDNA3.1COTE1-Flag were resuspended in $1 \mathrm{ml}$ lysis buffer $(20 \mathrm{mM}$ Tris, $\mathrm{pH}$ 7.5, $150 \mathrm{mM} \mathrm{NaCl}, 1.0 \%$ Triton X-100, 1 mM EDTA, and protease inhibitor cocktail). Immunoprecipitation of lysates was conducted using anti-Flag antibody (1:100; Santa Cruz Biotechnology), followed by immunoblotting with antibodies against WWOX (1:200; Santa Cruz Biotechnology) or COTE1 (1:100; Santa Cruz Biotechnology). Lysate of cells transfected with empty vector (pcDNA3.1) served as a control (12). 
A

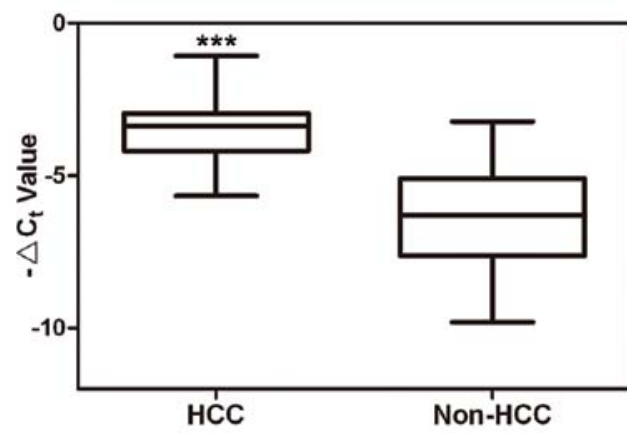

C

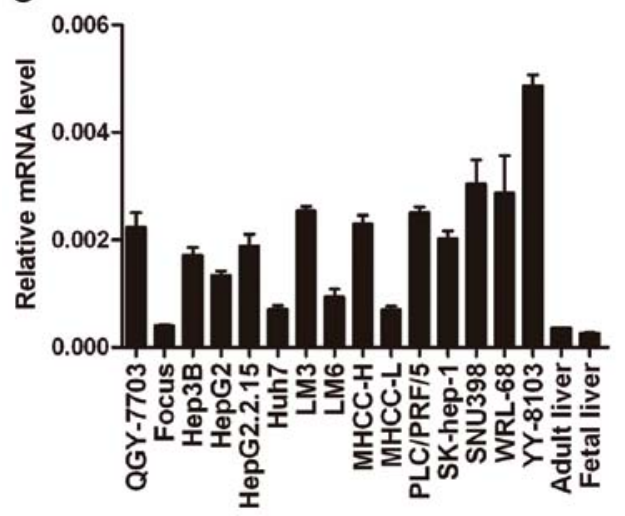

B

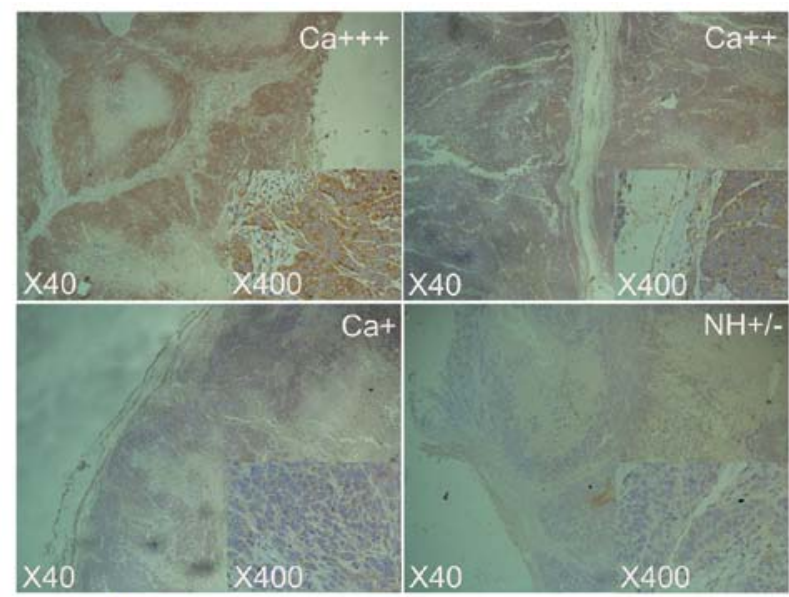

D

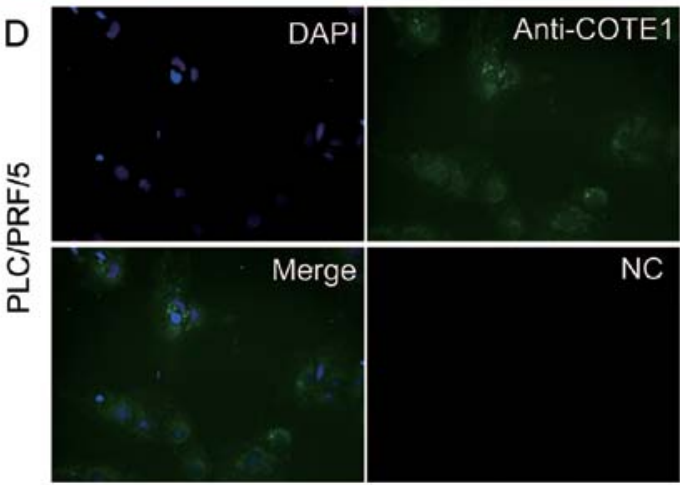

Figure 1. Expression pattern of COTE1 in HCC tissue specimens and cell lines. (A) Comparison of COTE1 mRNA expression in 80 paired HCC and non-HCC specimens by real-time PCR. The line within each box represents the median $\Delta \mathrm{C}_{\mathrm{t}}$ value; the upper and lower edges of each box represent the 75 th and 25 th percentile, respectively; the upper and lower bars indicate the highest and lowest values detected, respectively. For each sample, the relative COTE1 mRNA level was normalized to that of $\beta$-actin ${ }^{* * * *} \mathrm{p}<0.001$, Student's t-test). (B) Representative immunohistochemical staining of 80 paired HCC specimens and noncancerous tissue using anti-COTE1 antibody. The nuclei were counterstained with hematoxylin. The bottom right panel is enlarged from the center region of the middle image. Original magnification, $\mathrm{x} 40$ (middle); $\mathrm{x} 400$ (bottom right). $\mathrm{Ca}^{+++}$, cancerous specimen with strongly positive COTE1 expression; $\mathrm{Ca}^{++}$, cancerous specimen with moderately positive COTE1 expression; $\mathrm{Ca}^{+}$, cancerous specimen with mildly positive COTE1 expression; NH, non-HCC tissue with no or weakly positive expression of COTE1. (C) Expression of COTE1 in 15 HCC cell lines, healthy adult liver, and fetal liver was evaluated by real-time PCR, including $\beta$-actin as an internal control. (D) Cytoplasmic localization of endogenous COTE1 (green) in PLC/PRF/5 cells detected by immunofluorescent staining. The nuclei were stained with DAPI. Original magnification, $\mathrm{x} 400$.

Statistical analysis. All quantitative data were recorded as means \pm standard deviation (SD). Differences between two groups were assessed by Student's t-test (two-tailed) using GraphPad PRISM 5 software. Comparisons among multiple groups were performed by one-way analysis of variance and least significant difference t-test. Categorical data were evaluated by the $\chi^{2}$ test. In all tests, $\mathrm{p}<0.05$ was considered statistically significant.

\section{Results}

Overexpression of COTE1 and its association with malignancy of $H C C$. Previous gene microarray analysis performed in our laboratory showed that COTEl was upregulated in 11 paired HCC tissues (data not shown). To confirm these findings, we performed RT-PCR and quantitative PCR to measure the mRNA expression level of COTE1 in 80 paired HCC clinical specimens relative to the levels in corresponding adjacent non-cancerous liver. The results clearly showed upregulation of COTE1 mRNA in HCC specimens (Fig. 1A). To evaluate the protein level of COTE1 in HCC, we performed immunochemical staining with a specific antibody against COTE1 in another 80 matched samples. In non-HCC tissues 24/80 (30\%) showed no or weak (+/-) positive staining with the rest showed no staining, whereas 37/80 (46.25\%) of cancer specimens showed positive staining: seven mildly positive, 17 moderately positive, and 13 strongly positive (Fig. 1B). We also evaluated the expression pattern of COTE1 in HCC cell lines by RT-PCR and found that COTE1 was highly expressed in 11 of $15 \mathrm{HCC}$ cells relative to expression in normal human adult liver tissue (Fig. 1C). Together, these data showed that COTE1 is upregulated in $\mathrm{HCC}$, confirming the results of the gene microarray analysis.

To investigate the relationship between COTE1 expression and HCC clinical features, we further analyzed the results of RT-PCR in the 80 HCC specimens. The clinical characteristics of the patients and tumors are summarized in Table II. The prepared specimens were grouped by gender (male or female), age ( $\geq 45$ years or $<45$ years), etiology $\left(\mathrm{HBV}^{+}\right.$or $\left.\mathrm{HBV}^{-}\right)$, tumor size ( $\geq 3 \mathrm{~cm}$ or $<3 \mathrm{~cm}$ ), metastasis (yes or no) and Edmondson grade (I-II or III-IV). The resulting data showed that increased transcriptional expression of COTE1 was statistically correlated with the pathological tumor size and Edmondson grade $(\mathrm{p}<0.05)$. However, no statistical correlation was found for the other vari- 
Table II. Expression of COTE1 versus clinical features.

\begin{tabular}{|c|c|c|c|c|c|}
\hline HCC parameters & No. of patients & COTE1(+) $(\%)$ & COTE1(-) (\%) & $\chi^{2}$ & $\mathrm{p}$-value \\
\hline \multicolumn{6}{|l|}{ Gender } \\
\hline Male & 69 & $36 \quad(45.0)$ & $33(41.25)$ & 0.021 & 0.884 \\
\hline Female & 11 & $6 \quad(7.5)$ & $5 \quad(6.25)$ & & \\
\hline \multicolumn{6}{|l|}{ Age (years) } \\
\hline$\geq 45$ & 58 & $32(40.0)$ & $26 \quad(32.5)$ & 0.604 & 0.437 \\
\hline$<45$ & 22 & $10 \quad(12.5)$ & $12 \quad(15.0)$ & & \\
\hline \multicolumn{6}{|l|}{ Etiology } \\
\hline $\mathrm{HBV}^{+}$ & 67 & $36 \quad(45.0)$ & $31(38.75)$ & 0.251 & 0.617 \\
\hline $\mathrm{HBV}^{-}$ & 13 & $6 \quad(7.5)$ & $7 \quad(8.75)$ & & \\
\hline \multicolumn{6}{|c|}{ Pathological size $(\mathrm{cm})$} \\
\hline$\geq 3$ & 25 & $18 \quad(22.5)$ & $7 \quad(6.75)$ & 5.545 & 0.019 \\
\hline$<3$ & 55 & $24 \quad(30.0)$ & $31(38.75)$ & & \\
\hline \multicolumn{6}{|l|}{ Metastasis } \\
\hline Yes & 36 & $24(42.11)$ & $12(21.05)$ & 0.132 & 0.716 \\
\hline No & 21 & 13 (22.87) & $8(14.04)$ & & \\
\hline \multicolumn{6}{|l|}{ Edmondson grade } \\
\hline I-II & 49 & $21(26.25)$ & $28 \quad(35.0)$ & 4.715 & 0.03 \\
\hline III-IV & 31 & $21(26.25)$ & $10 \quad(12.5)$ & & \\
\hline
\end{tabular}

COTE(+) indicates upregulation of COTE1 gene (>0.5-fold); COTE1(-) indicates no upregulation of COTE1.

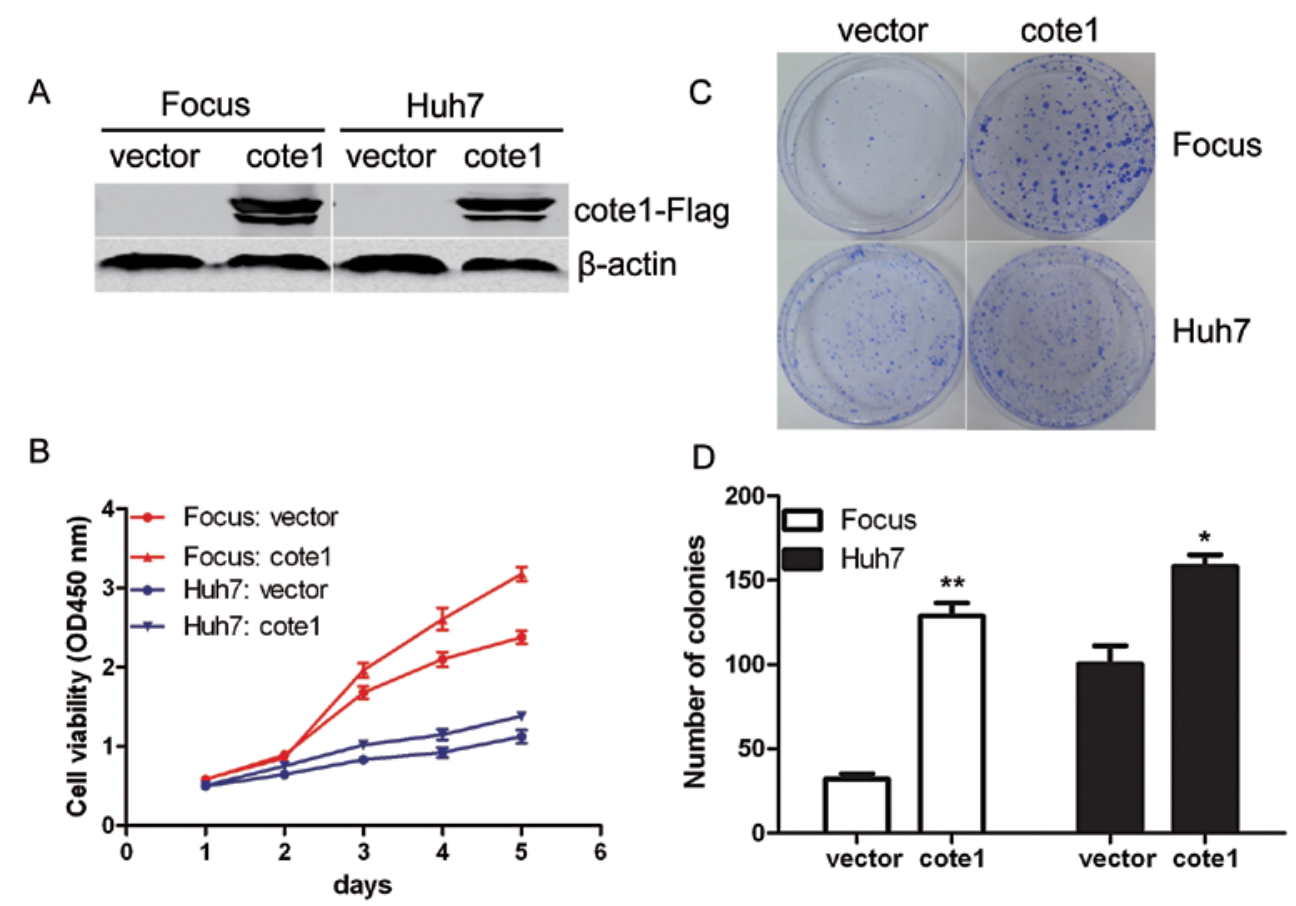

Figure 2. Exogenous COTE1 overexpression enhances cellular viability and colony formation. (A) Western blot analysis of exogenous COTE1 expression in Focus (left) and Huh7 (right) cells transfected with pcDNA3.1B-COTE1-Flag vector. Cells transfected with empty vector were used as a control. (B) Ectopic COTE1 expression promoted the proliferation of Focus (red) and Huh7 (blue) cells. Cell viability was analyzed using a CCK-8 kit. Three independent experiments were performed, and the symbols represent the mean values of triplicate tests $( \pm \mathrm{SD})$. There was a significant difference between the two groups ( $\mathrm{p}<0.05$, Student's t-test). (C) Overexpression of COTE1 by pcDNA3.1B-COTE1-Flag transfection enhanced colony formation of Focus (upper) and Huh7 (lower) cells. Each experiment was independently performed three times, and representative plates are shown. (D) Histograms showing colony number for empty vector and COTE1 transfectants; the numbers indicate the mean values of three independent experiments with SD. Colony formation of Focus and Huh7 cells was significantly enhanced by COTE1 ("p $<0.05,{ }^{* *} \mathrm{p}<0.01$, Student's t-test). 
A

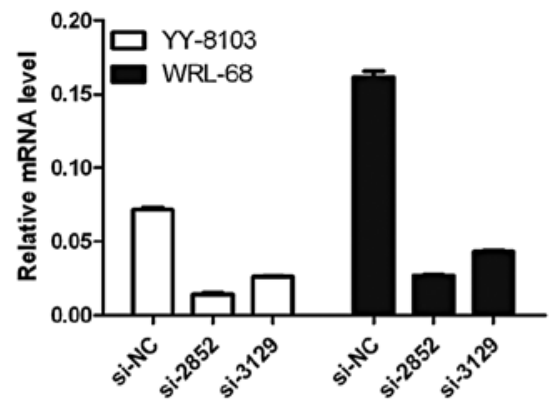

B

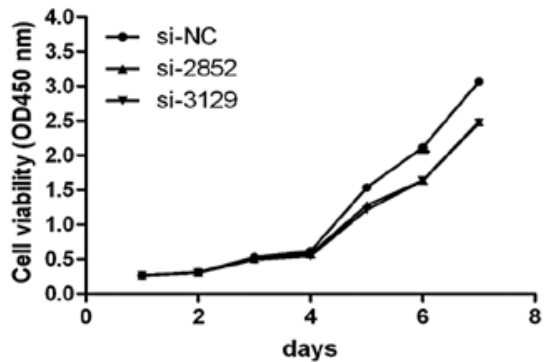

C

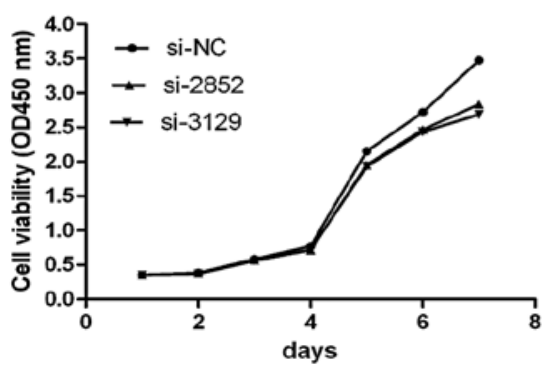

D

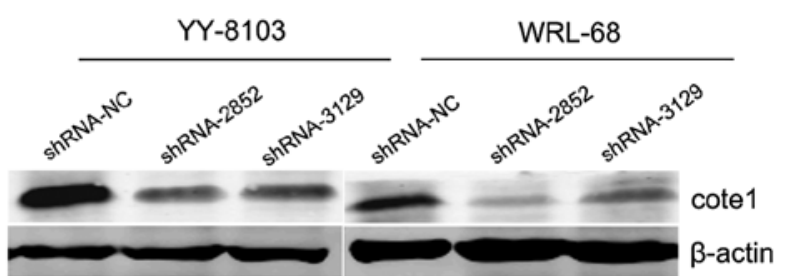

$\mathrm{E}$
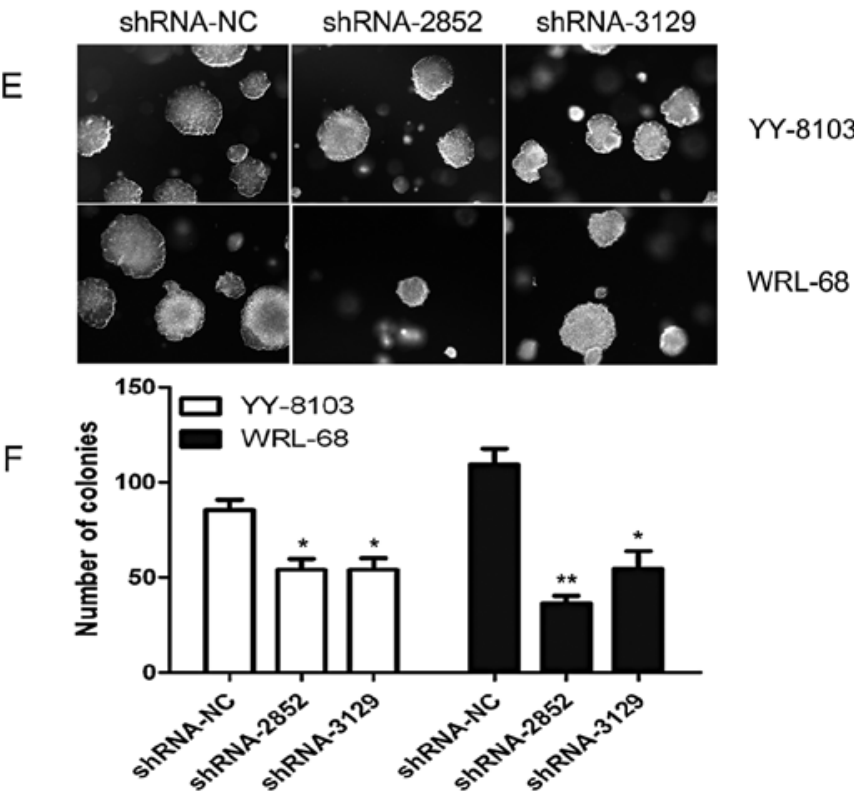

Figure 3. Silencing of COTE1 inhibits HCC cell proliferation and soft agar colony formation. (A) Endogenous COTE1 expression in YY-8103 and WRL-68 cells was knocked down by si-2852 and si-3129, as indicated by real-time PCR. (B, C) Cell growth of YY-8103 and WRL-68 transfected with COTE1 siRNA. Each symbol represents the mean value of triplicate experiments $( \pm$ SD). Compared with the negative control (si-NC), si-2852/3129 significantly suppressed the growth of YY-8103 (B) and WRL-68 (C) cells. (D, E, and F) Transfection with shRNA-2852/3129 inhibited colony formation of YY-8103 and WRL-68 cells in soft agar. (D) Western blot analysis confirming inhibition of COTE1 expression by the two shRNAs. (E) Representative results showing the decrease in anchorageindependent colony formation in cells transfected with COTE1 shRNA. The data in the histogram represent the means of three independent experiments $( \pm$ SD) $\left(\mathrm{p}<0.05,{ }^{* *} \mathrm{p}<0.01\right.$, compared with control). (F) Colony formation of cells transfected with shRNA, original magnification, $\mathrm{x} 100$.

ables. Taken together, these data indicate that upregulation of COTE1 contributes to HCC growth and poor differentiation.

COTE1 is localized in the cytoplasm and cell membrane. To identify the subcellular localization of COTE1 in HCC cells we performed immunofluorescence staining of endogenous COTE1 in PLC/PRF/5 cells. The results showed localization of COTE1 predominantly in the cytoplasm and to a lesser extent in the cell membrane of HCC cells (Fig. 1D).

Exogenous COTE1 promotes cellular proliferation and colony formation. To investigate whether COTE1 contributes to hepatocarcinogenesis, we investigated the effect of COTE1 on cell proliferation and colony formation. Based on the expression pattern of COTE1 in HCC cell lines, recombinant pcDNA3.1-COTE1-Flag was transiently transfected into Focus, Huh7, MHCC-LM6, and MHCC-L cells, all of which express a relatively low level of COTE1 (Fig. 1C). The empty vector pcDNA3.1-Flag was used as a control. Interestingly, cellular growth of Focus and Huh7 cells was significantly promoted by exogenous COTE1 compared with cells transfected with empty vector (Fig. 2A and B) but a similar response was not observed in MHCC-LM6 and MHCC-L. To further investigate the long- term effect of COTE1 on cell proliferation, transfected Focus and Huh7 cells were cultured in G418 for 2-3 weeks and colony formation was measured. Few colonies formed for cells transfected with vector alone, whereas colonies were more visible and a greater number of colonies formed in COTE1-overexpressing cells (Fig. 2C and D). These data suggest that ectopic COTE1 expression selectively enhanced the viability of HCC cells.

COTE1 knockdown inhibits cell growth and soft agar colony formation of HCC cells. We further evaluated the effect of COTE1 on cellular proliferation using YY-8103, WRL-68, PLC, and MHCC-97H cells, which showed high COTE1 expression levels (Fig. 1C). These cells were transfected with two chemically synthesized siRNAs that target COTE1, siRNA-2852 and siRNA-3129. As expected, endogenous COTE1 expression was efficiently knocked down in all cell lines. Proliferation was significantly inhibited in YY-8103 and WRL-68 cells transfected with siRNAs, but not in PLC or MHCC-97H cells (Fig. 3A-C). To further test the effect of COTE1 on cellular growth, we performed a soft agar colony formation assay, which more closely imitates in vivo growth. Given the short half-life of siRNAs, we used shRNAs derived from recombinant pSUPER vector for these experiments. YY-8103 and WRL-68 cells were 
A

Focus
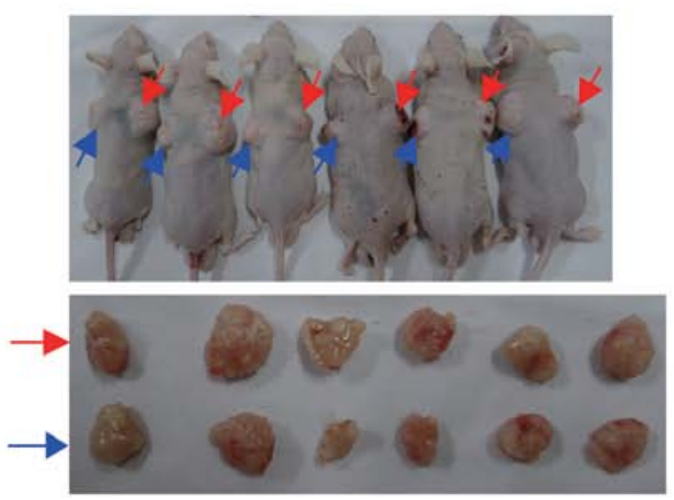

C
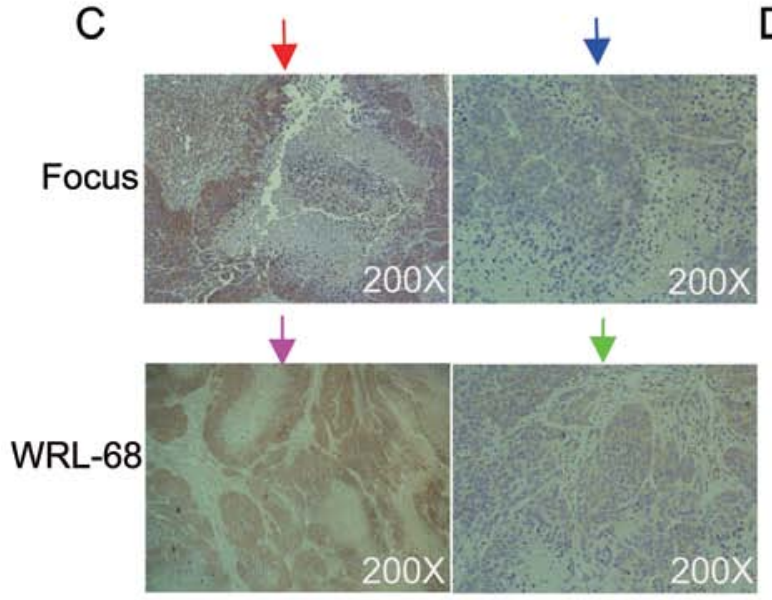

B
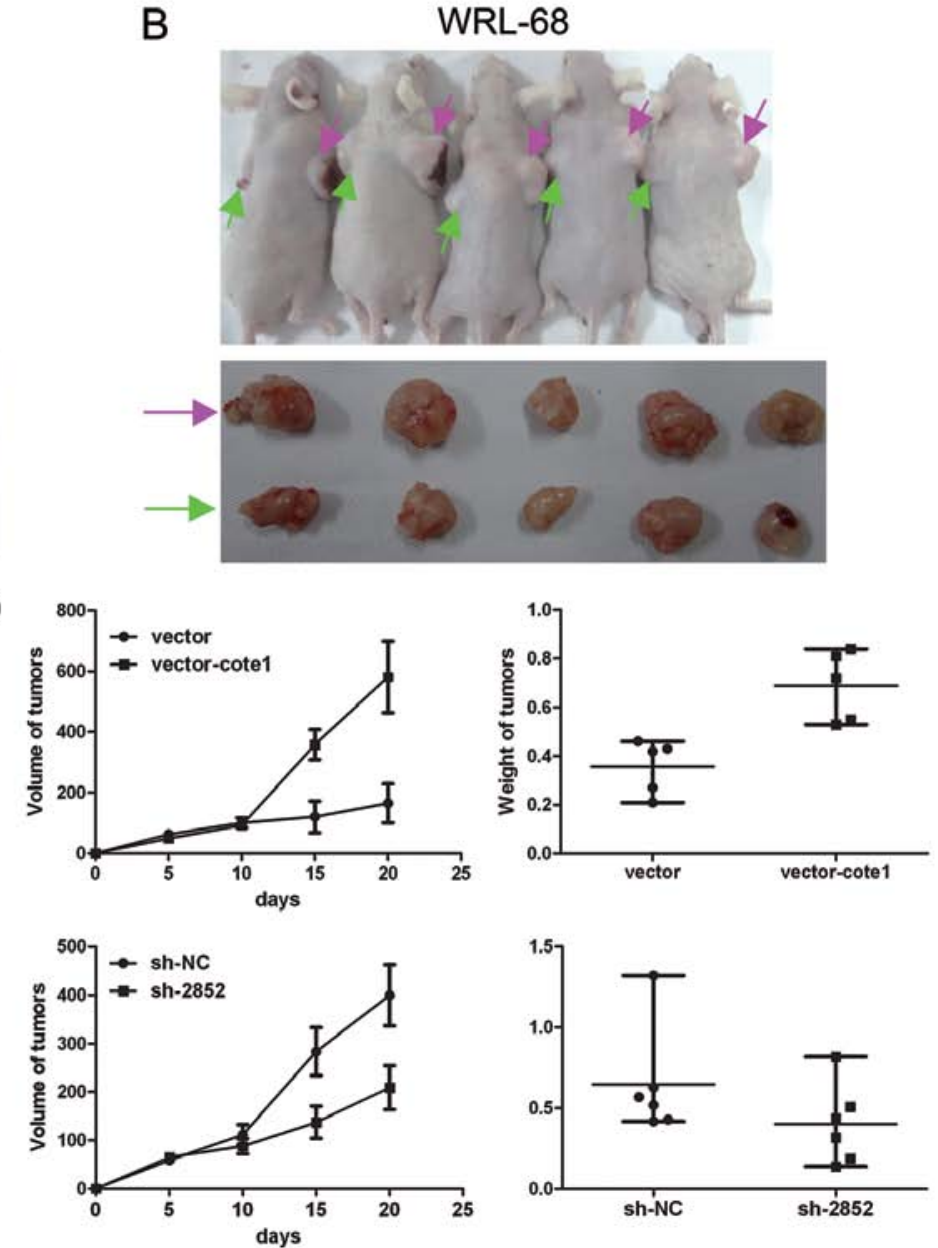

Figure 4. COTE1 promotes tumorigenicity of HCC cells. (A, B) Two stable transfected cell lines, Focus transfected with pcDNA3.1B-COTE1-Flag and WRL-68 transfected with shRNA-2852, were injected subcutaneously into mice. Overexpression of COTE1 enhanced tumorigenicity of Focus cells (A, upper), whereas gene silencing of COTE1 inhibited tumorigenicity of WRL-68 cells (B, upper). All xenograft tumors were removed and photographed after sacrificing the mice (A and B, lower). Red arrows, vector-COTE1; blue arrows, vector only; pink arrows, shRNA-NC; green arrows, shRNA-2852. (C) Immunohistochemical staining to verify the expression levels of COTE1 in xenografts. Focus cell tumors transfected with pcDNA3.1B-COTE1-Flag expressed high levels of COTE1, whereas WRL-68 tumors transfected with shRNA-2852 showed low levels of COTE1 expression. Original magnification, x200. (D) Growth of Focus (top left) and WRL-68 (bottom left) tumors was monitored for 5 days by measuring the diameter (mean \pm SD). Tumor weight of Focus (top right) and WRL-68 (bottom right) tumors was measured on the 20th day. Each scatter plot shows the weight of a given xenograft tumor, where the lines represent the median with interquartile range.

transfected with shRNA and cultured in soft agar for 2-3 weeks. As expected, cells transfected with shRNA-2852/3129 produced fewer colonies than those transfected with shRNA-NC plasmid (Fig. 3D-F). These collective data indicate that endogenous COTE1 plays an essential role in cellular proliferation and colony formation of HCC cells.

COTE1 contributes to the tumorigenicity of HCC in vivo. To assess the effect of COTE1 on HCC cell proliferation in vivo, stable COTE1-transfected Focus or WRL-68 cells were implanted into one flank of nude mice and negative control cells were injected into the other flank. As expected, transfection with pcDNA3.1B-COTE1-Flag markedly enhanced tumorigenicity of cells compared with the controls (Fig. 4A and D, upper), whereas shRNA2852 suppressed tumorigenicity (Fig. 4B and $\mathrm{D}$, lower). Immunohistochemical analysis confirmed elevated COTE1 expression in the tumors formed by COTE1-transfected cells, and reduced COTE1 expression in tumors formed by cells transfected with COTE1 shRNA (Fig. 4C).
Role of COTE1 in cell cycle regulation and apoptosis of HCC. We first performed flow cytometry to evaluate cell cycle distribution of Focus, Huh7, YY-8103, and WRL-68 cells 24 and $48 \mathrm{~h}$ after transfection with siRNA2852. G0/G1 phase arrest was obvious in YY-8103 cells $48 \mathrm{~h}$ after transfection (Fig. 5A and B). In contrast, G1- to S-phase transition was evident in Huh7 cells at $24 \mathrm{~h}$ and reached a peak at $48 \mathrm{~h}$ post-transfection (Fig. 5C and D).

We next performed flow cytometry and TUNEL assays to determine the effect of COTE1 on apoptosis under reduced serum conditions. First, we investigated the apoptosis of YY-8103 and WRL-68 cells at different time points after shRNA transfection. Compared with the control group, COTE1 inhibition resulted in a larger percentage of sub-G1 cells in WRL-68, but not YY-8103 cells, reaching a peak at $72 \mathrm{~h}$ (Fig. 6A). To obtain further evidence of apoptosis, we performed the TUNEL assay in WRL-68 cells at $72 \mathrm{~h}$ post-transfection and obtained results consistent with those of flow cytometry (Fig. 6B and C). The effect of COTE1 upregulation on apoptosis was examined by treatment of COTE1-overexpressing Focus and Huh7 cells with 

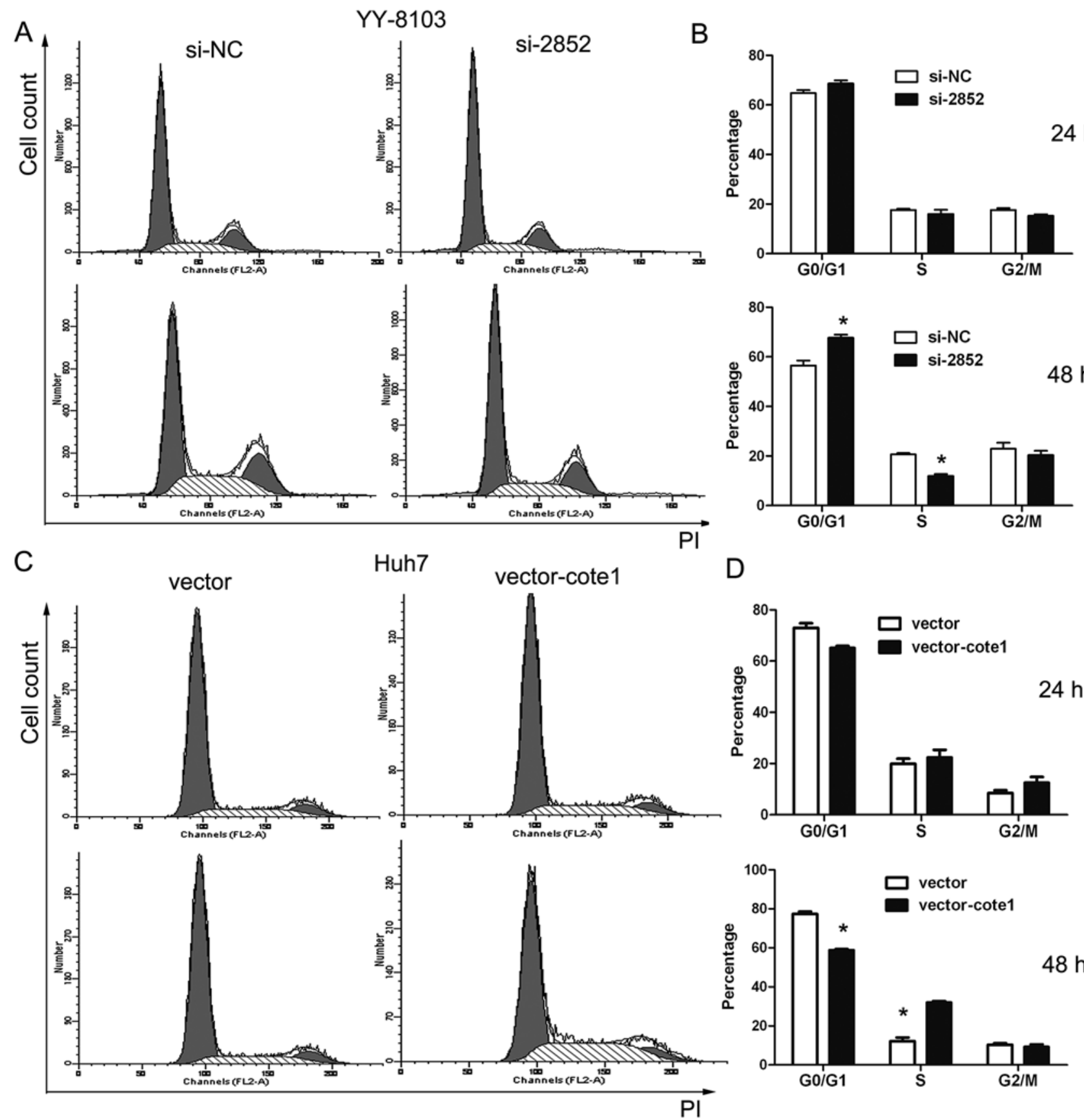

D
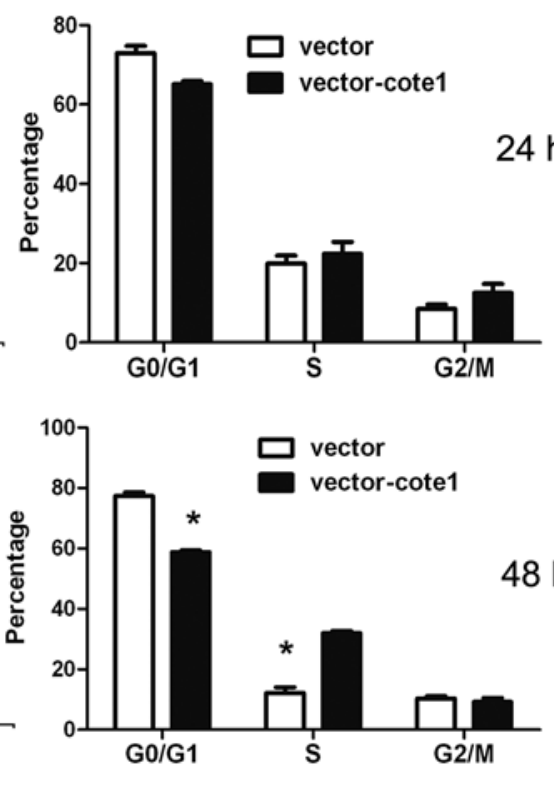

Figure 5. COTE1 participates in cell cycle regulation. (A) Flow cytometric analysis of cell cycle distribution in transfected HCC cells at 24 and $48 \mathrm{~h}$. (B) Histograms showing that COTE1 inhibition in YY-8103 cells delayed cell cycle progression via G0/G1 arrest at $48 \mathrm{~h}$. The experiment was repeated three times for each time point (mean $\pm \mathrm{SD})\left(\mathrm{p}<0.05,{ }^{* *} \mathrm{p}<0.01\right.$, compared with control). (C, D) COTE1 overexpression promotes cell cycle progression and suppresses doxorubicin-mediated apoptosis in HCC cells. COTE1 overexpression enhances cell cycle progression in Huh7 cells, with maximal effect at $48 \mathrm{~h}$ post-transfection (C). Histograms showing cell cycle distribution at $48 \mathrm{~h}$ post-transfection (D). The experiment was repeated three times at each time point (mean \pm SD; ${ }^{*}<<0.05$ ).

doxorubicin. As shown in Fig. 6D and E, doxorubicin $(5 \mu \mathrm{M}$, $24 \mathrm{~h}$ ) treatment resulted in a larger proportion of apoptotic cells in Focus cells transfected with empty vector compared with the COTE1 overexpressing cells.

COTE1 regulates the WWOX signaling pathway via inhibition of tyrosine phosphorylation. The data presented above suggest that COTE1 functions as a novel oncogene in HCC, since COTE1 inhibition obviously suppresses cell proliferation via cell cycle arrest and apoptosis. Considering previous reports that COTE1 binds to the WW domain of WWOX in vitro $(7,9)$, we investigated whether the effect of COTE1 on cell proliferation was mediated by WWOX. First, we carried out co-localization experiments to determine whether COTE1 might interact with WWOX, and observed COTE1-WWOX co-localization in the cytoplasm of Focus and Huh7 cells by fluorescence microscopy (Fig. 7A). Next, we performed co-immunoprecipitation (co-IP) assays to determine whether COTE1 and WWOX physically interact in Focus and Huh7 cells transfected with pcDNA3.1COTE1-Flag. The mutual co-IP data indicated that COTE1 physically associates with WWOX (Fig. 7B). To explore the effect of COTE1 on WWOX, we measured tyrosine phosphory- 
A
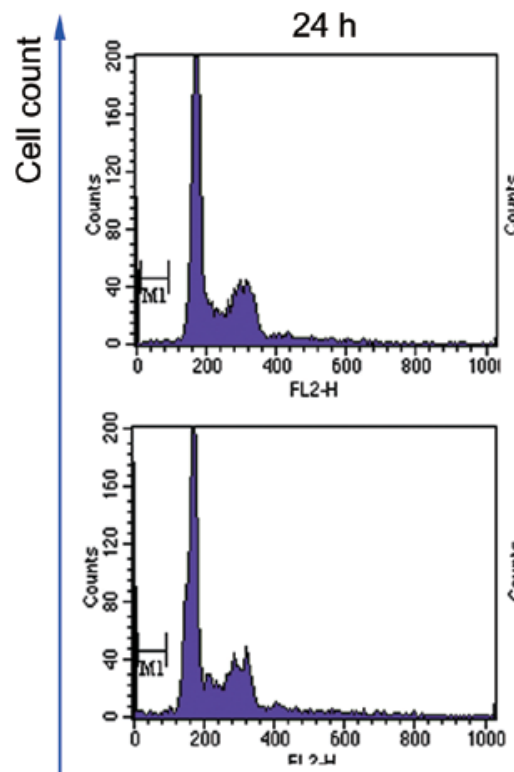

$48 \mathrm{~h}$
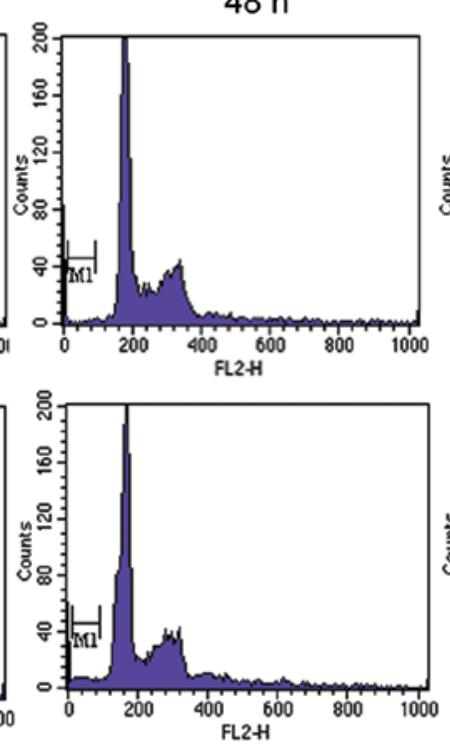

$72 \mathrm{~h}$
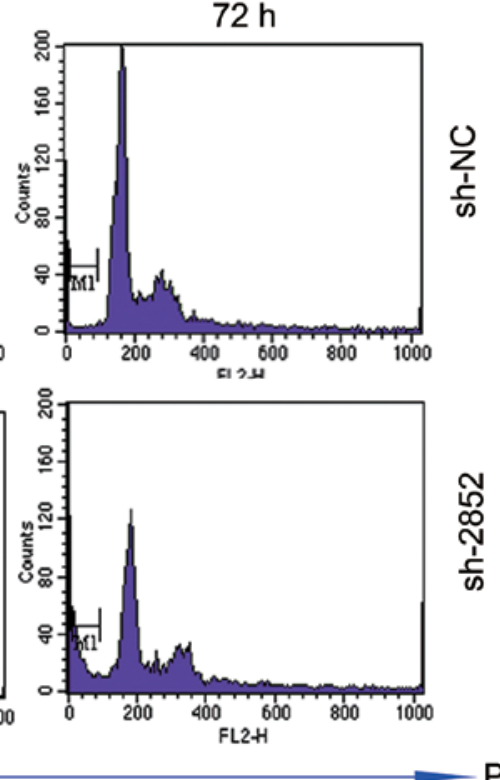

B

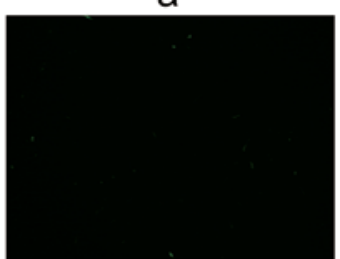

C

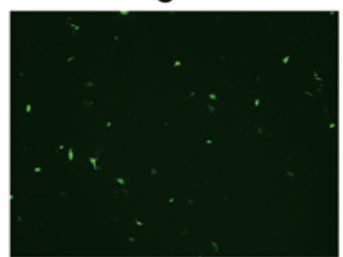

D

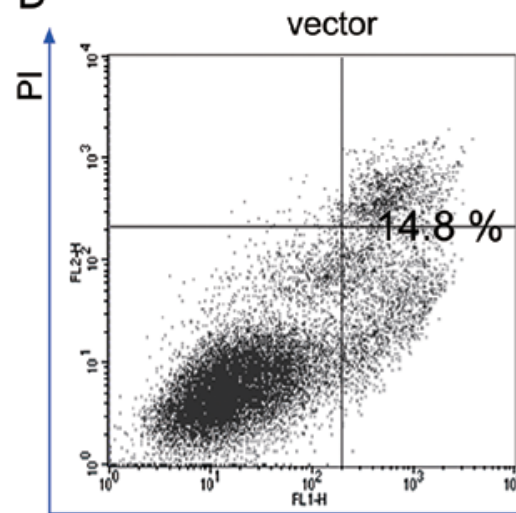

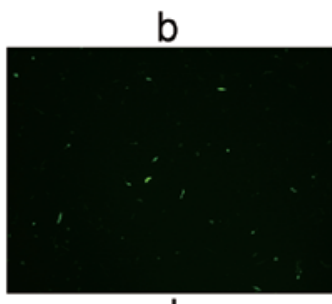

d

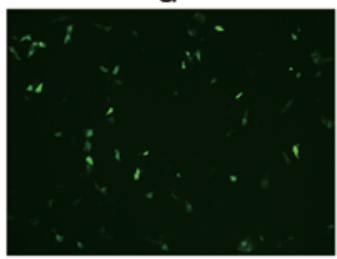

cote1

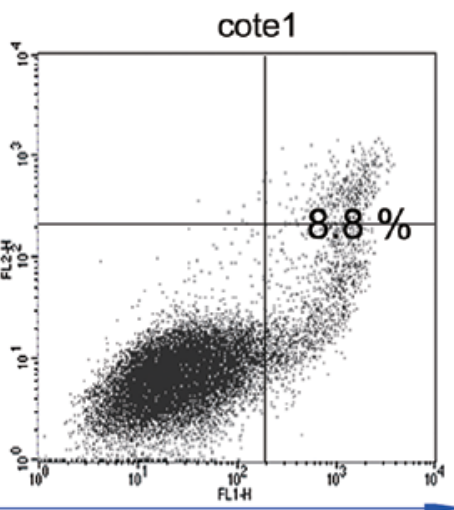

C

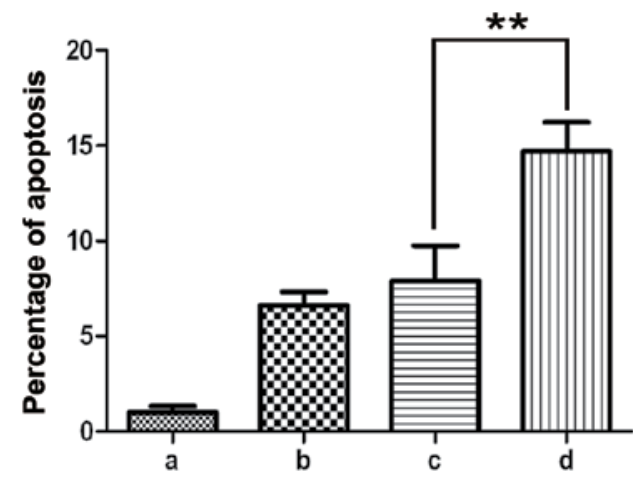

$\mathrm{E}$

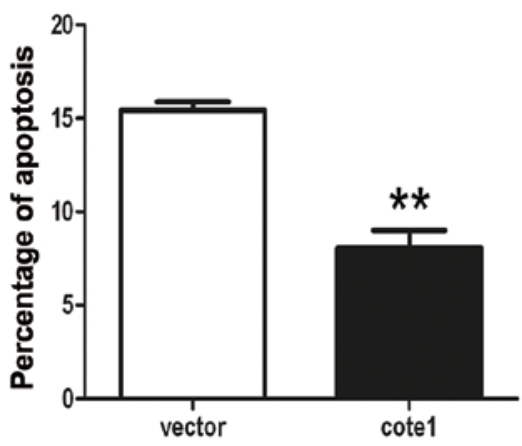

Annexin V FITC

Figure 6. COTE1 mediated apoptosis in HCC cells. (A, B, and C) Apoptosis in WRL-68 cells transfected with shRNA at 24, 48 and $72 \mathrm{~h}$ post-transfection. Cell apoptosis was assessed by flow cytometry (A) and TUNEL (B). Compared with the control group, the sub-diploid peak and the percentage of TUNEL-positive nuclei were significantly higher in cells transfected with COTE1-shRNA2852 (E, ${ }^{* *}$ p <0.01). a, null; b, lipofectamine 2000; c, lipofectamine 2000 + shNC; d, lipofectamine $2000+\operatorname{sh} 2852$. (D, E) Apoptosis in Focus cells transfected with vector alone or COTE1 and treated with doxorubicin $(5 \mu \mathrm{M}, 24 \mathrm{~h})$ analyzed by FACS with Annexin V/PI labeling. Data shown in the histogram (E) are the means of triplicate determinations $\left( \pm \mathrm{SD} ;{ }^{* *} \mathrm{p}<0.01\right)$.

lation of WWOX (Tyr33 and Tyr287) in transfected Focus, Huh7, YY-8103, and WRL-68 cells. Our data showed that the level of pTyr33 was decreased in Focus cells and increased in WRL-68; whereas the level of pTyr287 was reduced in Huh7 cells and increased in YY-8103 (Fig. 7C). We also measured p-p53 (Ser46), which participates in WWOX-mediated apoptosis, and found that p-p53 levels were suppressed in Focus cells, and increased in WRL-68 (Fig. 7C). 

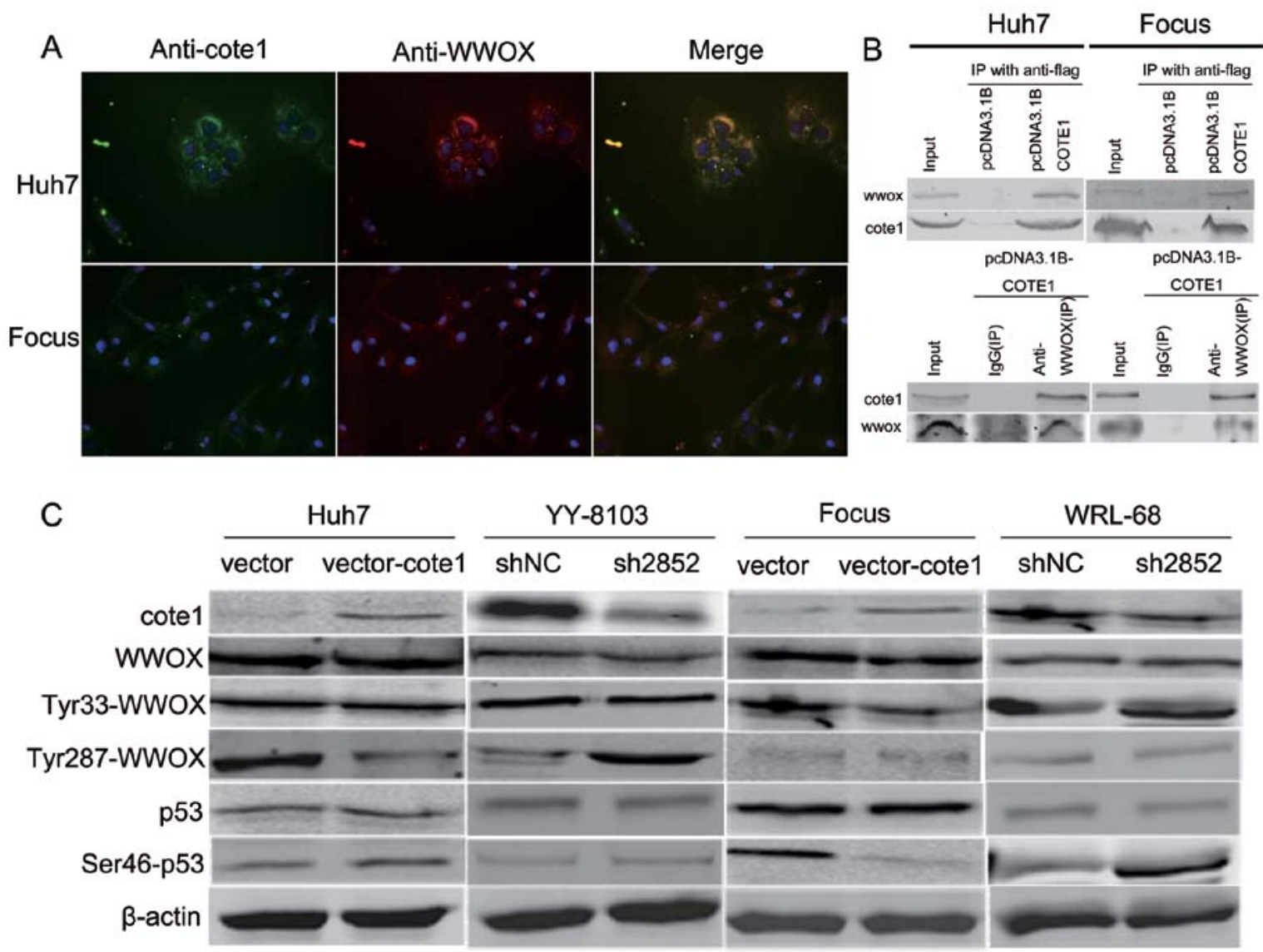

Figure 7. COTE1 physically interacts with WWOX and negatively regulates WWOX tyrosine phosphorylation. (A) Cytoplasmic colocalization of endogenous COTE1 (green) and WWOX (red) in Focus and Huh7 cells by immunofluorescence. Original magnification, x400. (B) Co-IP assays performed in Focus and Huh7 cells transfected with pcDNA3.1B-COTE1-Flag vector. Cells transfected with empty vector were used as a negative control. Endogenous WWOX was immunoprecipitated with the anti-Flag antibody (B, upper), and Flag-tagged COTE1 was reciprocally immunoprecipitated by the anti-WWOX antibody (B, lower). Native mouse IgG was used as the negative control, and residual total cell lysate was used for input. (C) Overexpression of COTE1 inhibited tyrosine phosphorylation of WWOX on Tyr33 in Focus cells and Tyr287 in Huh7 cells. COTE1 knockdown by shRNA promoted phosphorylation of Tyr33 in WRL-68 cells and Tyr287 in YY-8103 cells. COTE1 overexpression inhibited the activity of pp53 (Ser46) in Focus cells, but not in Huh7 cells. COTE1 knockdown by shRNA promoted the activity of pp53 (Ser46) in WRL-68, but not YY-8103, cells. The total levels of these proteins were assessed by immunoblotting with the corresponding antibodies. $\beta$-actin was used as a loading control.

COTE1-pWWOX mediates mitochondrial apoptosis and cell cycle regulation. To validate the hypothesis that COTE1pWWOX mediates mitochondrial apoptosis in HCC cells, we performed western blot analysis to measure the expression of molecules involved in the mitochondrial apoptosis pathway: Bax, Bcl-2, caspase-9, and caspase-3. Our data showed that upregulation of pWWOX (Tyr33) by COTE1 knockdown induced p53 (Ser46)-mediated endogenous apoptosis of WRL-68 cells and, conversely, Tyr33 dephosphorylation of WWOX by COTE1 overexpression rendered Focus cells resistant to p53 (Ser46)mediated apoptosis (Fig. 8A).

We then measured expression of cyclin D1 and cyclin E1, both of which function as positive regulators in promoting G1to S-phase transition, in Huh7 and YY-8103 cells and found that expression of cyclin D1, but not cyclin E1, negatively correlated with COTE1 expression. However, there was a positive correlation between cyclin D1 and pWWOX (Tyr287) (Fig. 8B). These data suggest that COTE1-pWWOX (Tyr287)cyclin D1 mediate cell cycle regulation in HCC.

In conclusion, the above findings indicate that COTE1 regulates the WWOX-p53-mediated apoptosis pathway through
Tyr33 dephosphorylation and participates in WWOX-induced cell cycle progression via Tyr287 dephosphorylation (Fig. 8C).

\section{Discussion}

Most cancer cells contain chromosomes that are broken, truncated, deleted, amplified, or translocated to other chromosomes. Such chromosomal abnormalities may lead to the inactivation of tumor suppressor genes or the activation of oncogenes via amplification (13). A previous study showed a high incidence of Clq copy number gain in HCC (60-80\%) (14). Many cancerrelated genes that are located at 1q12-q22, such as JTB, SHC1, CCT3, and COPA, have been shown to be up-regulated in HCC (15). COTE1, a novel potential oncogene that was identified by our laboratory, is located at chromosome 1q 21. Thus, we hypothesized that the COTE1 gene could be a candidate HCC-specific molecular marker.

The biological functions of COTE1, especially in cancers, remain unclear. In the present study, we showed that COTE1 was upregulated in HCC cancer tissue compared with adjacent normal liver tissue, and statistically correlated with tumor size 
A

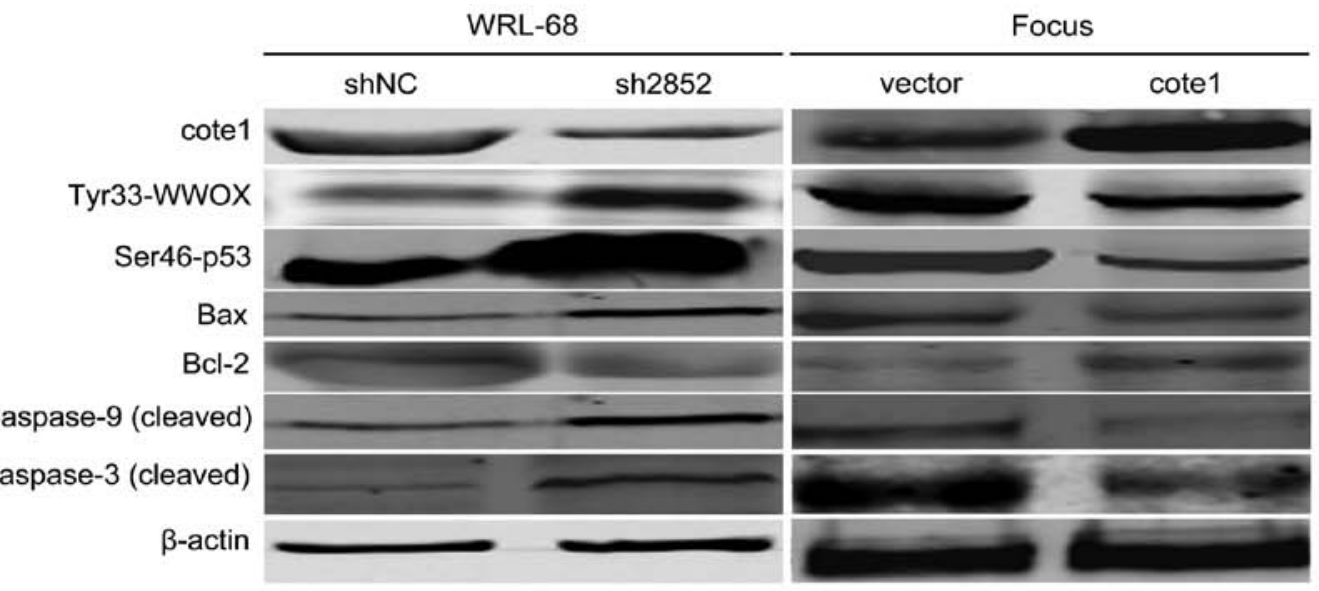
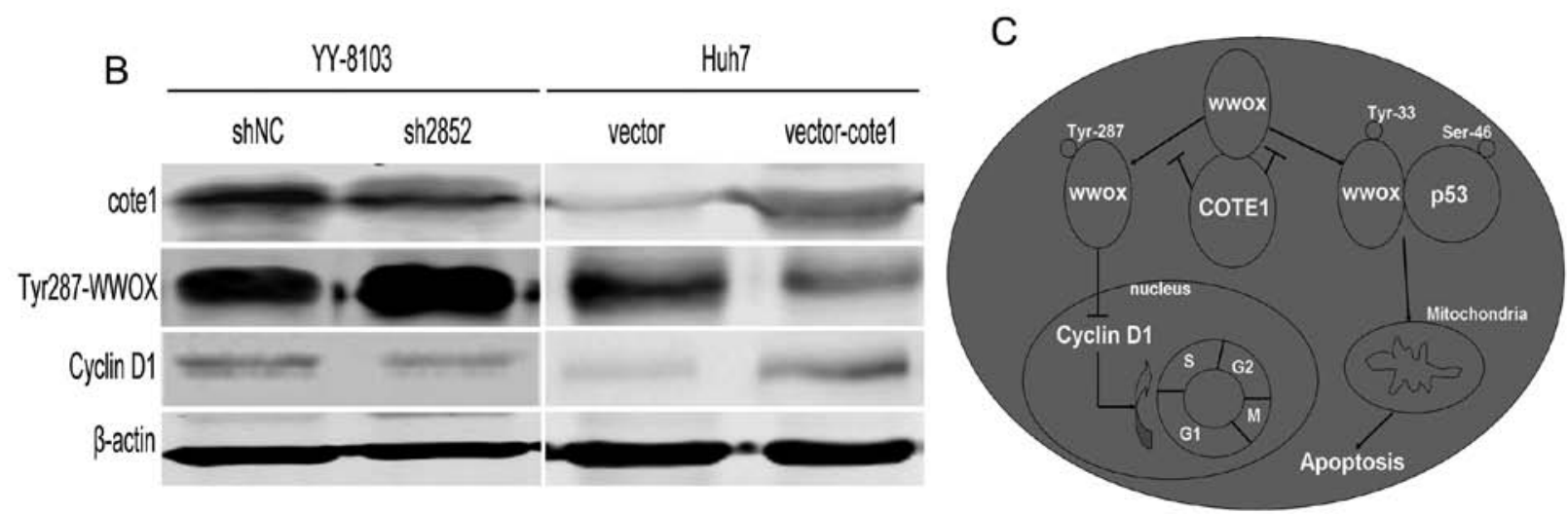

Figure 8. COTE1-pWWOX mediates cell cycle regulation and mitochondrial apoptosis of HCC cells. (A) Western blot analysis of endogenous apoptosis-associated downstream molecules, Bax, Bcl-2, caspase-9, and caspase-3, in COTE1 silenced WRL-68 cells and COTE1 overexpressing Focus cells. $\beta$-actin was used as a loading control. (B) Western blot analysis of cyclin D1 expression in COTE1 knockdown YY-8103 and COTE1 overexpressing Huh7 cells. $\beta$-actin was used as a loading control. (C) A hypothetical model of the functions of COTE1 in HCC via regulation of the WWOX signaling pathway. Arrows represent activation, whereas bars represent inhibition. In physiological conditions, COTE1 inhibits the tyrosine phosphorylation of WWOX by physically associating with WWOX, and thus blocks the WWOX signaling pathway. Upregulation of COTE1 in HCC may contribute to oncogenesis and tumor progression through multiple mechanisms. On the one hand, dephosphorylation of Tyr33 of WWOX by COTE1 results in decreased formation of WWOXTyr33-p53Ser46 complex, which in turn leads to mitochondrial apoptosis resistance. On the other hand, overexpression of COTE1 causes downregulation of WWOX Tyr287, which may negatively regulate the expression of cyclin D1 leading to cell cycle progression through promotion of the G1- to S-phase transition.

and differentiation. In addition, high expression of COTE1 was observed in HCC cell lines. These findings implied that COTE1 could function as an oncogene in HCC. We also showed that overexpression of COTE1 promoted proliferation of Focus and Huh7 cells in vivo and in vitro. In contrast, gene silencing of COTE1 reduced cell viability of YY-8103 and WRL-68 cells in vivo and in vitro. Together, these data suggest that COTE1 does indeed play an important role in HCC neoplasia. We subsequently revealed that knockdown of COTE1 induced cell cycle arrest in YY-8103 cells and apoptosis in WRL-68 cells. These data indicate that the oncogenic role of COTE1 in HCC is potentially mediated through regulation of the cell cycle and apoptosis.

COTE1 appears to participate in apoptosis regulation by direct physical association with the tumor suppressor WWOX and modulation of WWOX tyrosine phosphorylation. The WW domains of WWOX interact with a growing list of interesting proteins (16-18); for example, WWOX participates in TRADD (TNF receptor-associated death domain protein)-mediated cell death and mitochondrial apoptosis (19-23). WWOX is inactivated in a range of tumor cells, and its decreased activity correlates with the malignancy of human HCC and other tumors (24-28). Phosphorylation of WWOX at Tyr33 and subsequent phosphorylation of other focal apoptosis complex-associated proteins, such as p53, are required for mitochondrial apoptosis $(29,30)$. WWOX is typically localized in the mitochondria, nucleus, and Golgi $(31,32)$, and is released from mitochondria during the mitochondrial membrane permeability transition, when it translocates to the nucleus and cooperates with p53 to mediate apoptosis $(29,30,33)$. Surprisingly, our data indicated co-localization and co-immunoprecipitation of COTE1 and WWOX in HCC cells. We also found that COTE1 knockdown via RNAi stimulated the WWOX tyrosine phosphorylation cascade and apoptosisassociated downstream signaling pathways. This suggests that COTE1 contributes to HCC tumorigenesis by regulating the WWOX-p53-mediated endogenous apoptosis pathway through tyrosine-33 dephosphorylation of WWOX.

WWOX is also known to play an important role in the regulation of cell cycle progression $(29,34,35)$. It was previously shown that WWOX inhibits cell cycle progression (29) and that its expression levels are negatively correlated with 
the expression of cyclin D1 and E1 (35). Cyclin D1 and E1 are well-documented important regulators that promote the G1- to S-phase transition of the cell cycle and function as oncogenes in many cancers, including HCC (36-40). Here, we confirmed the negative correlation between COTE1 and cyclin D1 expression in Huh7 and YY-8103 cells, and further demonstrated that Tyr287 phosphorylation of WWOX is modulated by COTE1 in Huh7 and YY-8103 cells. However, no change in WWOX expression was detected. We speculate that phosphorylation of WWOX at Tyr287 modulates cell cycle progression by regulating the expression of cyclin D1. In addition, WWOX could be activated by phosphorylation of other sites, including Tyr61, Tyr293, and Ser14 residues $(29,30,41)$, that were not investigated in this study. Furthermore, the key domain of COTE1 responsible for tyrosine phosphorylation of WWOX remains unknown, and is worthy of in-depth research. The diverse ways in which COTE1 appears to contribute to HCC proliferation via different pathways encourages us to further explore the role of COTE1 in other HCC cell lines in future studies.

In conclusion, COTE1 contributes to neoplasia of HCC via WWOX-p53-mediated apoptosis and WWOX-cyclin D1 associated cell cycle delay regulation (Fig. 8C). Based on these findings, COTE1 may represent a new target for $\mathrm{HCC}$ gene therapy.

\section{Acknowledgments}

The authors thank Qing Deng for kindly providing us with the pSUPER-GFP and pcDNA3.1B-Flag-hrGFP plasmids and Qun Wang for her excellent technical assistance. This work was supported by the National Natural Science Foundation, China (grant no. 81170415).

\section{References}

1. Parkin DM, Bray F, Ferlay J and Pisani P: Global cancer statistics, 2002. CA Cancer J Clin 55: 74-108, 2005.

2. Wang JS, Huang T, Su J, et al: Hepatocellular carcinoma and aflatoxin exposure in Zhuqing Village, Fusui County, People's Republic of China. Cancer Epidemiol Biomarkers Prev 10: 143-146, 2001.

3. Coleman WB: Mechanisms of human hepatocarcinogenesis. Curr Mol Med 3: 573-588, 2003.

4. Aravalli RN, Steer CJ and Cressman EN: Molecular mechanisms of hepatocellular carcinoma. Hepatology 48: 2047-2063, 2008.

5. Yu W, Andersson B, Worley KC, et al: Large-scale concatenation cDNA sequencing. Genome Res 7: 353-358, 1997.

6. Winfield SL, Tayebi N, Martin BM, Ginns EI and Sidransky E: Identification of three additional genes contiguous to the glucocerebrosidase locus on chromosome 1q21: implications for Gaucher disease. Genome Res 7: 1020-1026, 1997.

7. Ludes-Meyers JH, Kil H, Bednarek AK, Drake J, Bedford MT and Aldaz CM: WWOX binds the specific proline-rich ligand PPXY: identification of candidate interacting proteins. Oncogene 23: 5049-5055, 2004.

8. Kallin A, Johannessen LE, Cani PD, et al: SREBP-1 regulates the expression of heme oxygenase 1 and the phosphatidylinositol-3 kinase regulatory subunit p55 $\gamma$. J Lipid Res 48: 1628-1636, 2007.

9. Abdeen SK, Salah Z, Maly B, et al: Wwox inactivation enhances mammary tumorigenesis. Oncogene 30: 3900-3906, 2001.

10. Zhang H, Huang CJ, Tian Y, Wang YP, Han ZG and Li XC: Ectopic overexpression of COTE1 promotes cellular invasion of hepatocellular carcinoma. Asian Pac J Cancer Prev 13: 5799-5804, 2012.

11. Ren F, Wu H, Lei Y, et al: Quantitative proteomics identification of phosphoglycerate mutase 1 as a novel therapeutic target in hepatocellular carcinoma. Mol Cancer 9: 81, 2010.
12. Huang J, Zheng DL, Qin FS, et al: Genetic and epigenetic silencing of SCARA5 may contribute to human hepatocellular carcinoma by activating FAK signaling. J Clin Invest 120: 223-241, 2010.

13. Kim TM, Yim SH, Shin SH, et al: Clinical implication of recurrent copy number alterations in hepatocellular carcinoma and putative oncogenes in recurrent gains on 1q. Int J Cancer 123: 2808-2815, 2008.

14. Wong N, Lam WC, Lai PB, Pang E, Lau WY and Johnson PJ: Hypomethylation of chromosome 1 heterochromatin DNA correlates with q-arm copy gain in human hepatocellular carcinoma. Am J Pathol 159: 465-471, 2001.

15. Wong N, Chan A, Lee SW, et al: Positional mapping for amplified DNA sequences on 1q21-q22 in hepatocellular carcinoma indicates candidate genes overexpression. J Hepatol 38: 298-306, 2003.

16. Aqeilan RI, Donati V, Gaudio E, et al: Association of Wwox with ErbB4 in breast cancer. Cancer Res 67: 9330-9336, 2007.

17. Del Mare S, Salah Z and Aqeilan RI: WWOX: its genomics, partners, and functions. J Cell Biochem 108: 737-745, 2009.

18. Kurek KC, Del Mare S, Salah Z, et al: Frequent attenuation of the WWOX tumor suppressor in osteosarcoma is associated with increased tumorigenicity and aberrant RUNX2 expression. Cancer Res 70: 5577-5586, 2010.

19. Chang NS, Pratt N, Heath J, Schultz L, Sleve D, Carey GB and Zevotek N: Hyaluronidase induction of a WW domain-containing oxidoreductase that enhances tumor necrosis factor cytotoxicity. J Biol Chem 276: 3361-3370, 2001.

20. Aqeilan RI, Donati V, Palamarchuk A, Trapasso F, et al: WW domain-containing proteins, WWOX and YAP, compete for interaction with ErbB-4 and modulate its transcriptional function. Cancer Res 65: 6764-6772, 2005.

21. Aderca I, Moser CD, Veerasamy M, et al: The JNK inhibitor SP600129 enhances apoptosis of HCC cells induced by the tumor suppressor WWOX. J Hepatol 49: 373-383, 2008.

22. Hong Q, Sze CI, Lin SR, et al: Complement Clq activates tumor suppressor WWOX to induce apoptosis in prostate cancer cells. PLoS One 4: e5755, 2009.

23. Teng CC, Yang YT, Chen YC, Kuo YM and Sze CI: Role of WWOX/WOX1 in Alzheimer's disease pathology and in cell death signaling. Front Biosci (Elite Ed) 4: 1951-1965, 2012.

24. Park SW, Ludes-Meyers J, Zimonjic DB, Durkin ME, Popescu NC and Aldaz CM: Frequent downregulation and loss of WWOX gene expression in human hepatocellular carcinoma. Br J Cancer 91: 753-759, 2004.

25. Nunez MI, Ludes-Meyers J, Abba MC, et al: Frequent loss of WWOX expression in breast cancer: correlation with estrogen receptor status. Breast Cancer Res Treat 89: 99-105, 2005.

26. Aqeilan RI and Croce CM: WWOX in biological control and tumorigenesis. J Cell Physiol 212: 307-310, 2007.

27. Ramos D, Abba M, López-Guerrero JA, et al: Low levels of WWOX protein immunoexpression correlate with tumour grade and a less favourable outcome in patients with urinary bladder tumours. Histopathology 52: 831-839, 2008.

28. Gourley C, Paige AJ, Taylor KJ, et al: WWOX gene expression abolishes ovarian cancer tumorigenicity in vivo and decreases attachment to fibronectin via integrin alpha3. Cancer Res 69: 4835-4842, 2009.

29. Chang NS, Doherty J and Ensign A: JNK1 physically interacts with WW domain-containing oxidoreductase (WOX1) and inhibits WOX1-mediated apoptosis. J Biol Chem 278: 9195-9202, 2003.

30. Chen ST, Chuang JI, Cheng CL, Hsu LJ and Chang NS: Lightinduced retinal damage involves tyrosine 33 phosphorylation, mitochondrial and nuclear translocation of WW domain-containing oxidoreductase in vivo. Neuroscience 130: 397-407, 2005.

31. Yang G, Zhang G, Pittelkow MR, Ramoni M and Tsao H: Expression profiling of UVB response in melanocytes identifies a set of p53-target genes. J Invest Dermatol 126: 2490-2506, 2006.

32. Gaudio E, Palamarchuk A, Palumbo T, Trapasso F, Pekarsky Y, Croce CM and Aqeilan RI: Physical association with WWOX suppresses c-Jun transcriptional activity. Cancer Res 66: 11585-11589, 2006.

33. Chang NS, Doherty J, Ensign A, Schultz L, Hsu LJ and Hong Q: WOX1 is essential for tumor necrosis factor-, UV light-, staurosporine-, and p53-mediated cell death, and its tyrosine 33-phosphorylated form binds and stabilizes serine 46-phosphorylated p53. J Biol Chem 280: 43100-43108, 2005. 
34. Salah Z, Aqeilan R and Huebner K: WWOX gene and gene product: tumor suppression through specific protein interactions. Future Oncol 6: 249-259, 2010

35. Płuciennik E, Nowakowska M, Wujcicka WI, Sitkiewicz A, Kazanowska B, Zielińska E and Bednarek AK: Genetic alterations of WWOX in Wilms' tumor are involved in its carcinogenesis. Oncol Rep 28: 1417-1422, 2012.

36. Nishida N, Fukuda Y, Komeda T, et al: Amplification and overexpression of the cyclin D1 gene in aggressive human hepatocellular carcinoma. Cancer Res 54: 3107-3110, 1994.

37. Deane NG, Parker MA, Aramandla R, et al: Hepatocellular carcinoma results from chronic cyclin D1 overexpression in transgenic mice. Cancer Res 61: 5389-5395, 2001.
38. Tashiro E, Tsuchiya A and Imoto M: Functions of cyclin D1 as an oncogene and regulation of cyclin D1 expression. Cancer Sci 98: 629-635, 2007.

39. Geng Y, Yu Q, Sicinska E, Das M, et al: Cyclin E ablation in the mouse. Cell 114: 431-443, 2003.

40. Hwang HC and Clurman BE: Cyclin E in normal and neoplastic cell cycles. Oncogene 24: 2776-2786, 2005.

41. Chang JY, He RY, Lin HP, et al: Signaling from membrane receptors to tumor suppressor WW domain-containing oxidoreductase. Exp Biol Med 235: 796-804, 2010 